\title{
ROSAT OBSERVATIONS OF NON-MAGNETIC CVS
}

\author{
A. VAN TEESELING ${ }^{1}$, F. VERBUNT ${ }^{2}$, K. BEUERMANN ${ }^{1}$ \\ 1. Universitäts-Sternwarte Göttingen, Germany \\ 2. Astronomical Institute, Utrecht, The Netherlands
}

\section{Introduction}

In non-magnetic cataclysmic variables the accreted matter forms an accretion disk around the white dwarf. In the boundary layer between the white dwarf and the accretion disk the accreted matter decelerates from Keplerian velocities to the rotation velocity of the white dwarf. If the accretion rate is high the boundary layer would be optically thick and cool $\left(T \sim 10^{5} \mathrm{~K}\right)$, and if the accretion rate is low the boundary layer would be optically thin and hot $\left(T \sim 10^{8} \mathrm{~K}\right)$ (Pringle \& Savonije 1979).

There are several observational problems with this simple picture: a soft $\mathrm{X}$-ray component could only be detected so far in 5 dwarf novae in outburst and not in any nova-like variable. Also in high-accretion-rate systems there is a hot optically thin X-ray source, which has, however, an X-ray luminosity which is much less than the UV luminosity of the system (van Teeseling \& Verbunt 1994). Finally, there is evidence for orbital modulation in the X-rays from some systems (e.g. van Teeseling et al. 1995).

We have analysed ROSAT PSPC observations of more than 40 non-magnetic cataclysmic variables (van Teeseling, Beuermann \& Verbunt 1996). With this large sample we could investigate how these observations constrain theoretical models for the X-ray source in non-magnetic cataclysmic variables. Here we summarize some results.

\section{Results}

Fig. 1 shows that the $\mathrm{X}$-ray count ratios of non-magnetic cataclysmic variables form a narrow band. The count ratios are consistent with absorbed optically thin spectra. Bremsstrahlung temperatures, inferred from fits to the ROSAT spectra, have been plotted against the UV+optical luminosity in Fig. 2. There appears to be no correlation between the temperature and the mass accretion rate in the system. This is not consistent with simple boundary-layer theories (Pringle \& Savonije 1979; Tylenda 1981).

There is a significant anti-correlation between the inferred emission mea273

A. Evans and J. H. Wood (eds.), Cataclysmic Variables and Related Objects, 273-276. (1) 1996 Kluwer Academic Publishers. Printed in the Netherlands. 


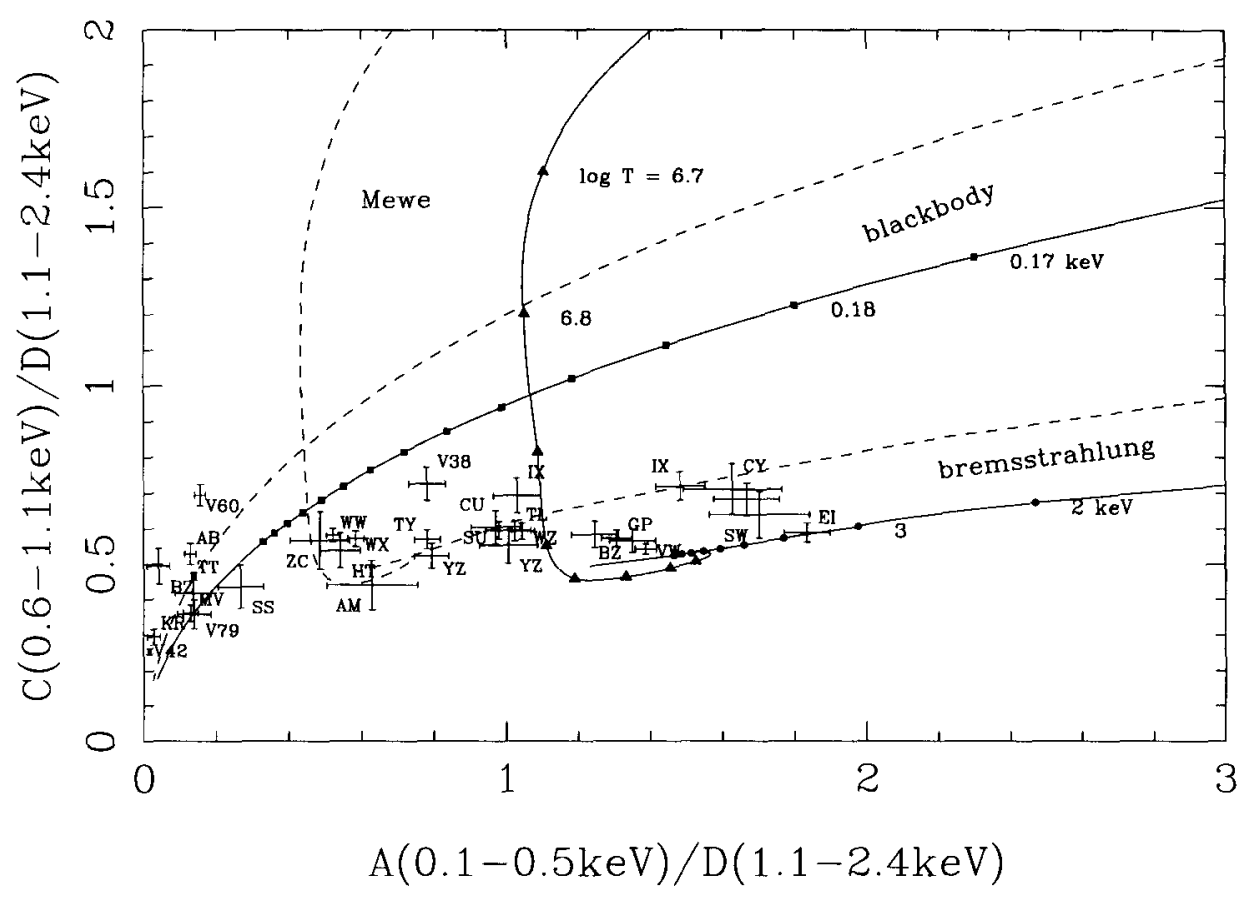

Figure 1. ROSAT PSPC count ratios of non-magnetic cataclysmic variables, with 1- $\sigma$ error bars. Systems are identified with the first letters of their name. Also plotted are the predicted count ratios for Kaastra \& Mewe (1993) spectra, blackbody spectra and thermal bremsstrahlung spectra. The theoretical spectra form bands with the solid boundary corresponding to zero interstellar absorption and the dashed boundary corresponding to a hydrogen column density of $n_{\mathrm{H}}=10^{20} \mathrm{~cm}^{-2}$. Absorption moves the predicted spectra almost horizontally to the left. The difference (in $\mathrm{keV}$ or $\log T$ ) between two subsequent marked points is constant. The highest blackbody temperature is $10 \mathrm{keV}$ and the highest bremsstrahlung temperature is $90 \mathrm{keV}$.

sure (or count rate $\times$ distance $^{2}$ ) (EM) and the orbital inclination. This is illustrated in Fig. 3, which contains 2 UX UMa systems, 2 Z Cam systems, 2 VY Scl systems, one non-SU UMa system, and 9 SU UMa systems. Fig. 3 also contains the candidate DQ Her systems TT Ari, SW UMa, WZ Sge, and V426 Oph. The anti-correlation in Fig. 3 implies that the $\mathrm{X}$-ray sources in all these systems are of similar nature. We can exclude all models in which the X-rays are emitted in a relatively large, optically thin volume. An optically thin boundary layer which is partly obscured by an optically thick inner accretion disk would have $\mathrm{EM} \propto 1 / \tan i$. Just as an illustration we have plotted the relation $\mathrm{EM}=210^{54} / \tan i \mathrm{~cm}^{-3}$.

For high- $\dot{M}$ systems the 'bolometric' X-ray flux is much lower than the UV+optical flux (Fig. 2). The X-ray luminosity is independent of the accretion rate $\dot{M}$, while the UV luminosity increases with increasing $\dot{M}$. 


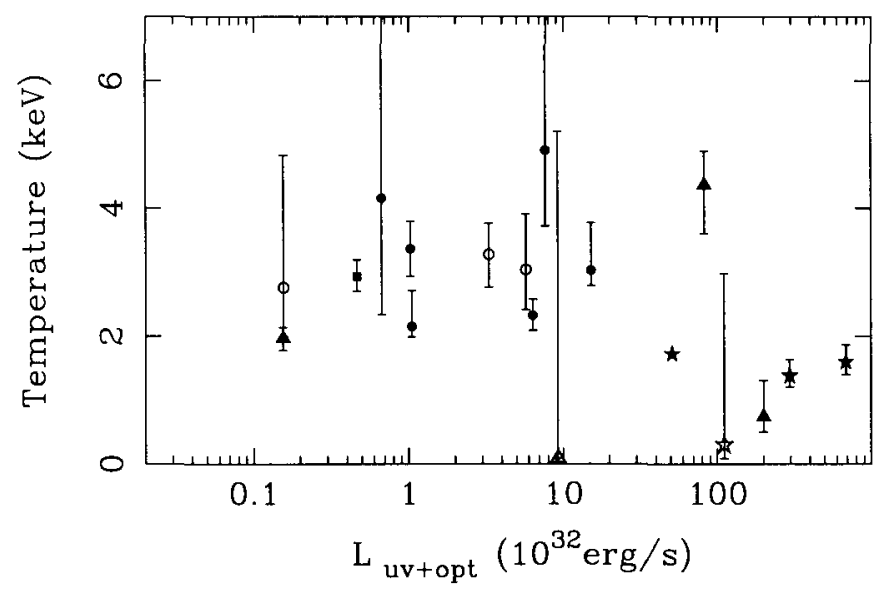

Figure 2. Bremsstrahlung temperature as a function of the ultraviolet+optical luminosity. Different symbols are used for subclasses: $\star=\mathrm{UX} \mathrm{UMa}, \bullet=\mathrm{SU}$ UMa, and $\star=$ other. For the open symbols the luminosity has been derived from the optical magnitude only.

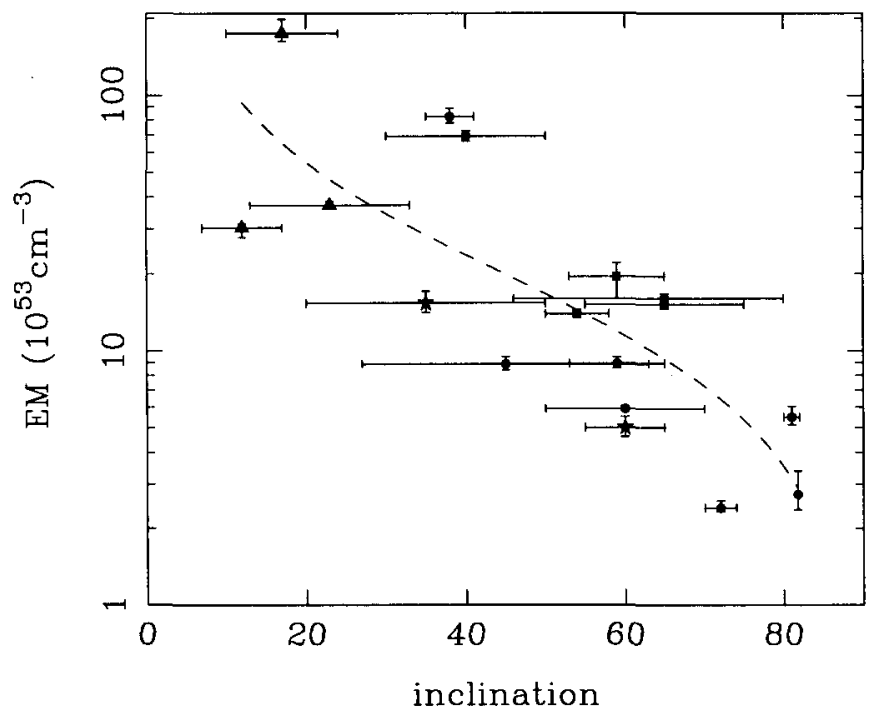

Figure 3. Observable emission measure as a function of the orbital inclination. The errors in the emission measures do not include the uncertainty in the distances. Different symbols are used for subclasses: $\star=\mathrm{UX} \mathrm{UMa}, \bullet=\mathrm{SU} \mathrm{UMa}, \boldsymbol{\bullet}=\mathrm{Z} \mathrm{Cam}$, and $\star=$ other. The dashed line is $\mathrm{EM}=210^{54} / \tan i \mathrm{~cm}^{-3}$. 


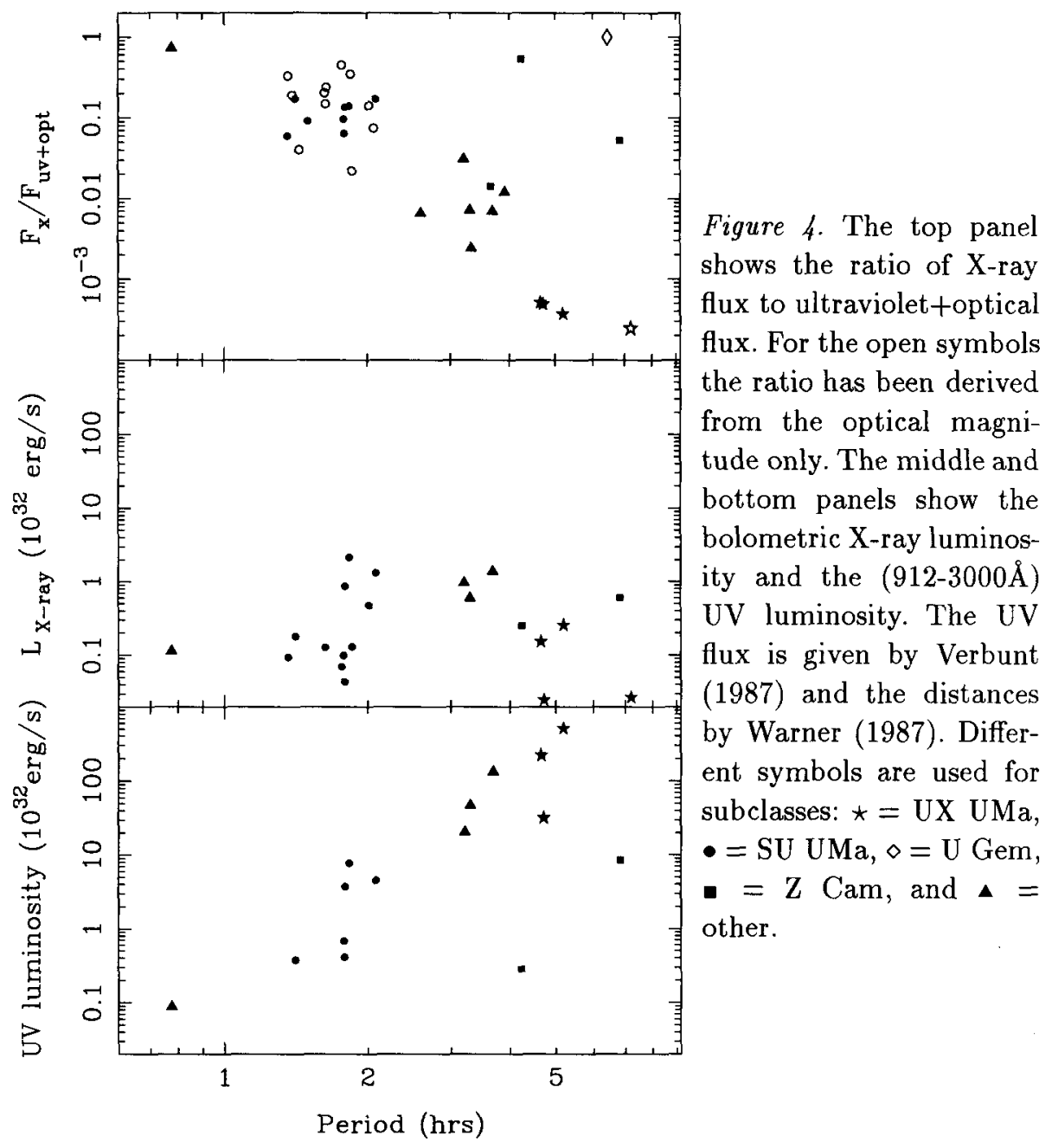

\section{References}

Kaastra, J.S., Mewe, R., 1993, Legacy, 3, 16

Pringle, J.E., Savonije, G.J., 1979, MNRAS, 187, 777

Tylenda, R., 1981, Acta. Astr., 31, 267

van Teeseling, A., Verbunt, F., 1994, A\&A, 292, 519

van Teeseling, A., Drake, J.J., Drew, J.E., et al., 1995, A\&A, 300, 808

van Teeseling, A., Beuermann, K., Verbunt, F., 1996, A\&A, submitted

Verbunt, F., 1987, A\&AS, 71, 339

Warner, B., 1987, MNRAS, 227, 23 